\title{
Prevalência de Transtornos Mentais Comuns e Fatores Associados em Estudantes de Medicina: um Estudo Comparativo
}

\author{
Prevalence of Common Mental Disorders \\ and Associated Factors among Medical \\ Students: a Comparative Study
}

\author{
Carlos Magno Guimarães Ferreira \\ Ana Claudia Garabeli Cavalli Kluthcovsky \\ Tatiana Menezes Garcia Cordeiro ${ }^{I}$
}

\section{PALAVRAS-CHAVE \\ - Transtornos Mentais; \\ - Estudantes de Medicina; \\ - Saúde Mental; \\ - Educação Médica.}

\section{KEYWORDS \\ - Mental Disorders; \\ - Medical Students; \\ - Mental Health; \\ - Medical Education.}

Recebido em: 13/11/2014

Aprovado em: 26/05/2016

\section{RESUMO}

Objetivos: Os objetivos do estudo foram estimar a prevalência de transtornos mentais comuns (TMC) e avaliar possíveis fatores associados em estudantes de Medicina de uma universidade pública do Sul do Brasil. Métodos: Estudo prospectivo com 134 estudantes de Medicina, comparando os resultados no início e final do semestre letivo. Para o rastreamento dos TMC utilizou-se o Self-Reporting Questionnaire. Também foram coletados dados socioeconômicos, demográficos, atividade física regular, doenças, atividades acadêmicas e qualidade do sono para avaliar possíveis associações com TMC. Resultados: A idade média foi de 22,5 anos ( $D P=4,3)$, a maioria do sexo masculino, solteiros e procedentes de outras cidades. A prevalência de TMC aumentou de 35,8\% no início para 51,5\% no final do semestre $(p=0,002)$. Os fatores associados aos TMC no início do semestre foram renda familiar mensal per capita $\leq R \$ 2.000,00(O R=3,2 ; I C 95 \%=1,31-8,02)$ e qualidade do sono ruim $(O R=3,3 ;$ IC95\% = 1,13-9,47). No final do semestre, a qualidade do sono ruim $(O R=3,3 ;$ IC $95 \%=$ 1,22-7,92) esteve associada aos TMC. Conclusão: Os resultados demonstram elevadas prevalências de TMC e a importância de direcionar ações de prevenção e enfrentamento ao cuidado com a saúde mental dos estudantes de Medicina.

\section{ABSTRACT}

Objective: The goal of this study was to estimate the prevalence of Common Mental Disorders $(C M D)$ in medical students from a university in the south of Brazil and to evaluate possible associated factors. Methods: The present study prospectively screened CMD using SRQ-20 in 134 medical students. Socioeconomic, demographic, lifestyle and health, academic activities and sleep quality data were also collected to evaluate possible associations with CMD. Results: The average age was 22.5 years (SD $=4.3)$, mostly male, single and from other cities. The prevalence of TMC increased from $35.8 \%$ at the beginning of the semester to $51.5 \%$ at the end of the semester $(p=0.002)$. Factors associated with CMD at the beginning of the semester were monthly per capita family income $\leq R \$$ $2,000.00(O R=3.2, I C 95 \%=1.31-8.02)$, and poor sleep quality $(O R=3.3, I C 95 \%=1.13-9.47) . A$ factor associated with CMD found at the end of the semester was poor sleep quality $(O R=3.3, I C 95 \%$ =1.22-7.92). Conclusion: The results showed a high prevalence of CMDs and the importance of targeting prevention and remedial actions at mental health care of medical students. 


\section{INTRODUÇÃO}

Faculdades de Medicina são reconhecidas como um ambiente estressante, que muitas vezes tem um efeito negativo sobre o desempenho acadêmico dos alunos, sua saúde física e bem-estar psicossocial ${ }^{1}$, podendo prejudicar sua qualidade de vida ${ }^{2}$. As atividades curriculares em horário integral e a realização de atividades complementares no curso de Medicina podem ocasionar desgaste psicológico, devido à pressão e estresse pelo alto rendimento, bem como à necessidade de maior tempo para os estudos ${ }^{3}$. Também pode contribuir para o aparecimento de depressão em estudantes uma variedade de fatores de estresse associados à formação profissional médica, como a pressão para aprender grande quantidade de novas informações, perda de oportunidades para atividades sociais e contato com doenças graves e com a morte no cuidado dos pacientes ${ }^{4}$.

Estudos vêm sendo realizados com a intenção de avaliar a saúde mental de estudantes da área da saúde ${ }^{1,4-8}$. Pesquisas também têm sido efetuadas com o propósito de verificar a prevalência de Transtornos Psiquiátricos Menores, também chamados Transtornos Mentais Comuns (TMC), em acadêmicos de Medicina ${ }^{9-18}$. No Brasil, um dos estudos pioneiros sobre o tema foi conduzido pela Universidade Federal de Santa Maria em $1996^{14}$, demonstrando uma prevalência de TMC de 31,7\%. Mais recentemente, um estudo desenvolvido com estudantes de Medicina em uma faculdade no Sudeste do Brasil estimou uma prevalência de $44,9 \%$.

Os TMC constituem sintomas não psicóticos, como dificuldade de concentração e tomada de decisões, irritabilidade, fadiga, sonolência, insônia, esquecimento, bem como queixas somáticas (tremores, cefaleia, má digestão, entre outros) ${ }^{19}$. Esses transtornos são extremamente onerosos para a qualidade de vida da pessoa e para os relacionamentos interpessoais ${ }^{9}$, além de potencial causa para o desenvolvimento de doenças mentais, como transtornos somatoformes, ansiedade e depressão ${ }^{20}$. Podem também estar associados a alterações do padrão do sono ${ }^{9,15}$ e a doenças crônicas ${ }^{21}$.

Diante do possível impacto negativo dos TMC na vida e no aprendizado dos estudantes e pela escassez de estudos comparativos sobre TMC, os objetivos deste estudo foram estimar a prevalência de TMC e avaliar possíveis fatores associados em estudantes de Medicina de uma universidade pública do Sul do Brasil, comparando os resultados obtidos no início e final do semestre letivo.

\section{MÉTODOS}

Tipo de estudo, local e sujeitos pesquisados

Estudo prospectivo e comparativo sobre TMC envolvendo estudantes do primeiro ao quarto ano do curso de Medicina, regularmente matriculados em uma universidade pública do Sul do Brasil. Trata-se de um curso realizado em período integral, com duração de seis anos. Optou-se por realizar a pesquisa com os estudantes do primeiro ao quarto ano do curso, pois estes estão passando pelas disciplinas de formação básica geral e de formação específica profissional, constituindo, portanto, um grupo mais homogêneo com relação ao cotidiano acadêmico se comparado ao período de internato, realizado no quinto e sexto anos do curso. Além disso, os alunos matriculados no internato realizam as atividades em vários estabelecimentos de saúde, fora das dependências do local proposto para o desenvolvimento da pesquisa (campus universitário), o que dificultaria localizá-los para a coleta de dados.

A universidade onde foi realizado o estudo se localiza em uma cidade de médio porte e é polo regional, sendo referência na região para as áreas de saúde e ensino. Oferece diversos cursos universitários em instituições tanto públicas quanto privadas, atraindo muitos estudantes de outras cidades, inclusive de outras regiões do País.

A coleta de dados foi realizada em sala de aula, em horário predeterminado, em dois momentos, no início e no final do semestre letivo, para fins de comparação. O primeiro momento ocorreu entre os meses de fevereiro e março de 2013. Como o período de férias escolares ocorreu na segunda quinzena de dezembro de 2012 e em janeiro de 2013, o primeiro momento de coleta de dados ocorreu logo após o período das férias escolares, para todos os alunos pesquisados. $\mathrm{O}$ segundo momento da coleta de dados ocorreu no final do semestre, no mês de junho de 2013.

Do total de 158 estudantes matriculados, 134 (84,8\%) participaram das duas etapas da pesquisa, sendo 31 do primeiro ano, 35 do segundo, 35 do terceiro e 33 do quarto ano do curso. Foram excluídos os estudantes com menos de 18 anos e aqueles que não estavam presentes no momento da coleta de dados em sala de aula e não foram encontrados em uma nova busca. Oito alunos recusaram participar.

\section{Instrumentos de coleta de dados}

Foram utilizados três questionários para coleta de dados, todos autoaplicáveis e anônimos.

O primeiro se referia a informações socioeconômicas e demográficas (ano do curso, idade, sexo, estado civil, com quem reside, cidade de procedência, renda familiar mensal per capita e independência financeira), atividades acadêmicas (realizar atividade extracurricular, fazer plantão, número de horas semanais dessas duas atividades e realizar atividade acadêmica remunerada), atividade física regular e presença de doença atual. 
Para avaliar possíveis casos de TMC, foi utilizado o Self-Reporting Questionnaire (SRQ-20). Esse questionário foi desenvolvido pela Organização Mundial da Saúde em 1980 para rastrear distúrbios psiquiátricos em nível de atenção primária $^{22}$ e validado no Brasil, com propriedades psicométricas satisfatórias ${ }^{23}$. O SRQ-20 é utilizado para o rastreamento de quatro fatores (humor depressivo/ansioso, sintomas somáticos, decréscimo da energia vital e pensamentos depressivos), conhecidos como distúrbios psiquiátricos menores ${ }^{24}$. É composto por 20 questões com respostas "sim" ou "não". Os sintomas avaliados se referem aos últimos 30 dias, e a cada resposta "sim" é atribuído um ponto, resultando em uma pontuação final entre 0 e 20. A pontuação total de TMC foi obtida pela soma de todos os itens do instrumento. Estudantes do sexo masculino com pontuação igual ou superior a 6 e do sexo feminino com pontuação igual ou superior a 8 foram classificados como possíveis casos de $\mathrm{TMC}^{22-23}$.

Para verificar uma possível associação de TMC com qualidade do sono, foi utilizado o Índice de Qualidade de Sono de Pittsburgh (PSQI-BR) ${ }^{25}$, traduzido e validado para o português ${ }^{26}$ e que avalia a qualidade do sono no período de um mês anterior à entrevista. O questionário contém 19 questões autoaplicáveis e cinco questões respondidas pelo companheiro de quarto. Estas últimas não foram utilizadas no presente estudo, pois são usadas somente para informação clínica. As questões têm pesos distribuídos em uma escala de 0 a 3 , onde 0 indica qualidade muito boa e 3 qualidade muito ruim de sono. Para a avaliação da qualidade do sono, as pontuações dos componentes do PSQI-BR foram somadas para se produzir um escore global entre 0 e 21. Estudantes com pontuação maior do que 5 tiveram sua qualidade de sono classificada como ruim, e estudantes com pontuação igual ou inferior a 5 tiveram sua qualidade de sono classificada como boa ${ }^{25-26}$.

\section{Análise estatística dos dados}

Estatísticas descritivas foram realizadas para apresentar as características dos participantes, os percentuais das respostas afirmativas do SRQ-20 e a prevalência dos TMC. Para as comparações dos dados socioeconômicos e demográficos entre o início e o final do semestre foi utilizado o teste de Qui-Quadrado de Pearson.

O teste de Qui-Quadrado de McNemar para amostras pareadas foi empregado para comparar as prevalências de TMC no início e final do semestre. O teste de Qui-Quadrado de Pearson foi usado para avaliar a associação das diversas variáveis com a variável de desfecho (possíveis casos ou não de TMC), para o início e final do semestre, em separado. As análises multivariadas foram conduzidas por meio de regressão lo- gística (método stepwise forward), para identificar as variáveis associadas com TMC. Foram incluídas no modelo as variáveis com valor de $\mathrm{p}<0,20$ na análise bivariada, calculando-se odds ratio e respectivos intervalos de confiança de $95 \%$.

Os dados foram processados em planilha eletrônica no Programa Microsoft Office Excel® 2010 para Windows ${ }^{\circledR}$, com dupla digitação para correção de erros de transcrição, quando necessário. A análise estatística foi obtida com auxílio do programa Statistical Package for Social Science (SPSS), versão 16.0. O nível de significância utilizado foi de $5 \%$.

O projeto foi aprovado pelo Comitê de Ética em Pesquisa da instituição de ensino sob o Parecer no 159.536 em 30 de novembro de 2012, CAAE: 11341212.4.0000.0105. Antes da coleta de dados, todos os estudantes foram informados sobre a pesquisa, seus objetivos e o sigilo quanto às respostas fornecidas. Todos os participantes assinaram o Termo de Consentimento Livre e Esclarecido, em duas vias.

\section{RESULTADOS}

A Tabela 1 apresenta as variáveis do ano do curso, socioeconômicas e demográficas no início do semestre, relacionadas aos 134 estudantes pesquisados. Comparando-se as variáveis socioeconômicas e demográficas no início e no final do semestre, nenhuma apresentou diferença significativa: idade $(p=0,125)$, estado civil $(p=1,000)$, com quem mora $(p=0,581)$, procedência $(p=1,000)$, renda familiar mensal per capita $(p=0,210)$ e independência financeira $(p=0,250)$.

A média de idade dos estudantes foi de 22,5 anos ( $\mathrm{DP}=$ $4,3)$ no início do semestre e 22,7 anos ( $D P=4,3)$ no final do semestre. Com relação à renda familiar mensal per capita, os menores valores relatados foram de $\mathrm{R} \$ 375,00$ e $\mathrm{R} \$ 300,00$ no início e final do semestre, respectivamente, e os maiores valores relatados foram $\mathrm{R} \$ 16.667,00$ e $\mathrm{R} \$ 20.000,00$ no início e final do semestre, respectivamente.

TABELA 1

Distribuição do total dos acadêmicos de Medicina segundo as variáveis ano do curso, socioeconômicas e demográficas no início do semestre $(n=134)$

\section{Variáveis}

Ano do curso

Primeiro

31

Segundo

Terceiro

Idade

Até 20 anos 43 


\begin{tabular}{|c|c|c|}
\hline $\begin{array}{l}\text { Distribuição do total } \\
\text { segundo as variáveis a } \\
\text { e demográficas no }\end{array}$ & $\begin{array}{l}\mathrm{Mc} \\
\text { eco } \\
\mathrm{n}=\end{array}$ & na \\
\hline Variáveis & $\mathbf{N}$ & $\%$ \\
\hline$>20$ anos & 90 & 67,7 \\
\hline Sexo & & \\
\hline Feminino & 64 & 47,8 \\
\hline Masculino & 70 & 52,2 \\
\hline Estado civil & & \\
\hline Solteiro(a) & 125 & 92,5 \\
\hline Casado(a) & 9 & 6,7 \\
\hline Com quem reside & & \\
\hline Sozinho(a) & 49 & 36,6 \\
\hline Familiares/colegas & 84 & 63,4 \\
\hline Procedência & & \\
\hline Cidade local & 40 & 29,9 \\
\hline Outra cidade & 94 & 70,1 \\
\hline Independência financeira & & \\
\hline $\begin{array}{l}\text { Não } \\
\text { Sim }\end{array}$ & $\begin{array}{r}130 \\
4\end{array}$ & $\begin{array}{r}97,0 \\
3,0\end{array}$ \\
\hline
\end{tabular}

Dados faltantes: estado civil $(n=1)$ e renda $(n=28)$.

* O valor de $R \$ 2.000,00$ era equivalente a aproximadamente 3 salários mínimos ou US\$ $896,06 \mathrm{em} \mathrm{01/062013.}$

\section{TABELA 2}

Proporções de respostas afirmativas das questões do SelfReporting Questionnaire (SRQ-20) para avaliar possíveis casos de Transtornos Mentais Comuns em estudantes de Medicina no início e final do semestre $(n=134)$

Respostas
Itens do SRQ-20 por grupos de sintomas

\section{afirmativas $(\%)$}

\begin{tabular}{ll}
\hline Início do & Final do \\
semestre & semestre
\end{tabular}

\section{Humor depressivo/ansioso}

Assusta-se com facilidade

$18,7 \quad 26,1$

Chora mais do que de costume

14,9

26,1

Sente-se triste ultimamente

14,2

20,1

\section{Sintomas somáticos}

Sente desconforto estomacal

38,8

43,3

Dorme mal

35,1

Má digestão

35,1

Dores de cabeça frequentes

30,6

Tremores nas mãos

Falta de apetite

23,9

21,6

25,4

Decréscimo de energia vital

Cansa-se com facilidade

Sente-se cansado o tempo todo

58,6

51,5

37,3

39,1

Dificuldade em tomar decisão

$\begin{array}{ll}58,6 & 65,7 \\ 44,0 & 55,2 \\ 35,1 & 43,3\end{array}$

TABELA 2

Proporções de respostas afirmativas das questões do Self-

Reporting Questionnaire (SRQ-20) para avaliar possíveis casos de Transtornos Mentais Comuns em estudantes de Medicina no início e final do semestre $(n=134)$

\begin{tabular}{ccc} 
Itens do SRQ-20 & \multicolumn{2}{c}{$\begin{array}{c}\text { Respostas } \\
\text { afirmativas (\%) }\end{array}$} \\
\cline { 2 - 3 } por grupos de sintomas & $\begin{array}{l}\text { Início do } \\
\text { semestre }\end{array}$ & $\begin{array}{l}\text { Final do } \\
\text { semestre }\end{array}$
\end{tabular}

$\begin{array}{lcc}\begin{array}{l}\text { Dificuldade de pensar claramente } \\ \begin{array}{l}\text { Dificuldade de ter satisfação em suas } \\ \text { tarefas }\end{array}\end{array} & 28,4 & 41,8 \\ \begin{array}{l}\text { O trabalho/ocupação traz sofrimento } \\ \text { Pensamentos depressivos }\end{array} & 15,7 & 23,1 \\ \text { Perdeu o interesse pelas coisas } & 15,7 & 23,1 \\ \text { Pensa em dar fim à vida } & 13,4 & 12,7 \\ \text { É difícil sentir-se útil/importante } & 5,2 & 8,2 \\ \text { Sente-se inútil/sem importância } & 4,5 & 8,2\end{array}$

A Tabela 2 apresenta as proporções de repostas afirmativas de cada questão do SRQ-20 utilizado para avaliar os TMC para o total de alunos pesquisados, agrupados por categorias, no início e final do semestre. Os maiores percentuais de respostas afirmativas foram para a questão sobre sentir-se nervoso, tenso ou preocupado, que passou de $71,6 \%$ no início do semestre para 78,4\% no final do semestre. Em seguida, estão as respostas sobre ficar cansado com facilidade, que passou de $58,6 \%$ para $65,7 \%$, se sentir cansado o tempo todo, de $44,0 \%$ para $55,2 \%$, e dormir mal, de $35,1 \%$ para $51,5 \%$ no início e final do semestre, respectivamente.

\begin{tabular}{|c|c|c|c|c|c|}
\hline \\
\hline \multirow{3}{*}{\multicolumn{6}{|c|}{$\begin{array}{l}\text { Distribuição das prevalências de Transtornos } \\
\text { Mentais Comuns para o total dos acadêmicos de } \\
\text { Medicina no início e no final do semestre }(n=134)\end{array}$}} \\
\hline & & & & & \\
\hline & & & & & \\
\hline \multirow{2}{*}{ TMC } & \multicolumn{2}{|c|}{ Início do semestre } & \multicolumn{2}{|c|}{ Final do semestre } & \multirow{2}{*}{$p^{*}$} \\
\hline & $\mathrm{n}$ & $\%$ & $\mathbf{n}$ & $\%$ & \\
\hline Positivo & 48 & 35,8 & $69^{* *}$ & 51,5 & 0,002 \\
\hline Negativo & 86 & 64,2 & $65^{* * *}$ & 48,5 & \\
\hline
\end{tabular}

* Teste McNemar para amostras pareadas.

** 32 desses casos eram TMC negativos no início do semestre.

*** 11 desses casos eram TMC positivos no início do semestre.

A prevalência de TMC para o total de alunos no início do semestre foi de $35,8 \%$ e no final do semestre aumentou para $51,5 \%$. A comparação para amostras pareadas da prevalência de TMC no início e final do semestre revelou uma diferença significativa, sendo maior no final do semestre $(p=0,002)$ (Tabela 3). 


\begin{tabular}{|c|c|c|c|c|c|c|}
\hline \multicolumn{7}{|c|}{$\begin{array}{l}\text { TABELA } 4 \\
\text { Associação de variáveis (socioeconômicas e demográficas, atividades acadêmicas, atividade física, } \\
\text { doenças atuais e qualidade do sono) com a ocorrência de Transtornos Mentais Comuns (SRQ- } \\
\text { 20) para o total de acadêmicos de Medicina no início e final do semestre }(\mathrm{n}=134)\end{array}$} \\
\hline \multirow[b]{2}{*}{ Variáveis } & \multicolumn{3}{|c|}{ Início do semestre } & \multicolumn{3}{|c|}{ Final do semestre } \\
\hline & $\begin{array}{c}\text { TMC } \\
\text { negativo } \\
(\%)\end{array}$ & $\begin{array}{c}\text { TMC } \\
\text { positivo } \\
(\%)\end{array}$ & $p$ & $\begin{array}{c}\text { TMC } \\
\text { negativo } \\
(\%)\end{array}$ & $\begin{array}{c}\text { TMC } \\
\text { positivo } \\
(\%)\end{array}$ & $p$ \\
\hline \multicolumn{7}{|l|}{ Socioeconômicas e demográficas } \\
\hline Idade (20 anos ou menos) & 31,4 & 33,3 & 0,818 & 26,2 & 31,9 & 0,466 \\
\hline Sexo feminino & 47,7 & 47,9 & 0,979 & 41,5 & 53,6 & 0,162 \\
\hline Estado civil solteiro & 94,1 & 93,8 & 0,932 & 92,2 & 94,1 & 0,660 \\
\hline Morar sozinho & 39,5 & 31,3 & 0,340 & 35,4 & 33,3 & 0,803 \\
\hline Procedência de outra cidade & 70,9 & 68,8 & 0,791 & 67,7 & 71,0 & 0,677 \\
\hline Renda familiar mensal per capita $\leq \mathrm{R} \$ 2.000,00^{*}$ & 50,7 & 69,2 & 0,063 & 64,0 & 60,7 & 0,718 \\
\hline Não ter independência financeira & 97,7 & 95,8 & 0,548 & 95,3 & 94,2 & 0,775 \\
\hline \multicolumn{7}{|l|}{ Atividades acadêmicas } \\
\hline Realizar atividade extracurricular & 76,7 & 87,5 & 0,131 & 76,9 & 91,3 & 0,022 \\
\hline Atividade extracurricular $>5 \mathrm{~h} /$ semana $^{* *}$ & 37,5 & 42,1 & 0,645 & 37,8 & 25,5 & 0,195 \\
\hline Fazer plantão & 22,1 & 29,8 & 0,326 & 21,5 & 29,0 & 0,322 \\
\hline Plantão > 10 h/semana** & 26,3 & 42,9 & 0,319 & 50,0 & 35,0 & 0,382 \\
\hline Não realizar atividade acadêmica remunerada & 69,8 & 81,3 & 0,147 & 70,3 & 75,4 & 0,513 \\
\hline Não realizar atividade física regular & 69,8 & 79,2 & 0,239 & 73,8 & 88,4 & 0,031 \\
\hline Apresentar doença atual & 24,4 & 35,4 & 0,176 & 16,9 & 34,8 & 0,019 \\
\hline Qualidade do sono ruim (PSQI) & 62,8 & 85,4 & 0,006 & 58,5 & 84,1 & 0,001 \\
\hline
\end{tabular}

Teste Qui-Quadrado.

TMC = Transtornos mentais comuns; PSQI = Índice de Qualidade de Sono de Pittsburgh

* Equivalente a aproximadamente 3 salários mínimos ou US\$ 896,06 em 01/062013.

** Responderam apenas os que realizavam a atividade no início e final do semestre, respectivamente: atividade extracurricular $(n=102, n=97)$ e plantão $(n=33, n=34)$.

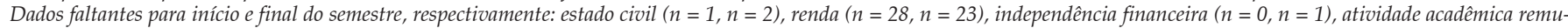

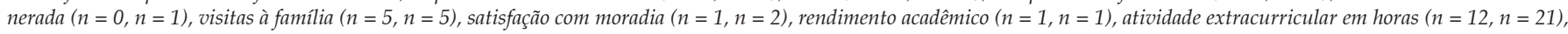
fazer plantão $(n=1, n=0)$, plantão em horas $(n=1, n=1)$.

As prevalências de TMC para cada ano do curso no início do semestre foram de $25,8 \%$ para o primeiro ano, $40 \%$ para o segundo, $43,2 \%$ para o terceiro e $33,3 \%$ para o quarto ano. Para o final do semestre, as prevalências foram de $41,9 \%, 62,9 \%$, $45,7 \%$ e $54,5 \%$, para o primeiro, segundo, terceiro e quarto anos, respectivamente. Na comparação das prevalências de TMC entre os estudantes dos quatro anos do curso, não se observou diferença significativa tanto para o início do semestre $(\mathrm{p}=0,482)$ como para o final $(\mathrm{p}=0,316)$.

A Tabela 4 mostra a análise bivariada entre TMC e as variáveis de interesse, tanto para o início como para o final do semestre. A variável que se apresentou significativa para TMC no início do semestre foi qualidade do sono ruim ( $p=0,006)$. No final do semestre, as variáveis significativas observadas foram: realizar atividades extracurriculares $(p,=, 0,022)$, não realizar atividade física regularmente $(p=0,031)$, apresentar doença atual $(p=0,019)$ e qualidade do sono ruim $(p=0,001)$.
Foram incluídas no modelo da análise multivariada, para o primeiro semestre, as seguintes variáveis: renda familiar mensal per capita $\leq \mathrm{R} \$ 2.000,00$, realizar atividade extracurricular, não realizar atividade acadêmica remunerada, apresentar doença atual e qualidade do sono ruim. Para o final do semestre, as variáveis foram: sexo feminino, realizar atividade extracurricular, atividade extracurricular maior que 5 horas por semana, não realizar atividade física regular, apresentar doença atual e qualidade do sono ruim.

$\mathrm{Na}$ análise da regressão logística, as variáveis associadas com os TMC que permaneceram significativas no início do semestre foram renda familiar mensal per capita $\leq \mathrm{R} \$ 2.000,00$ $(\mathrm{OR}=3,2$; IC95\% = 1,31-8,02) e qualidade do sono ruim (OR = 3,3; IC95\% = 1,13-9,47). Para o final do semestre, apresentou significância a qualidade do sono ruim (OR = 3,3; IC95\% = 1,22-7,92) (Tabela 5). 


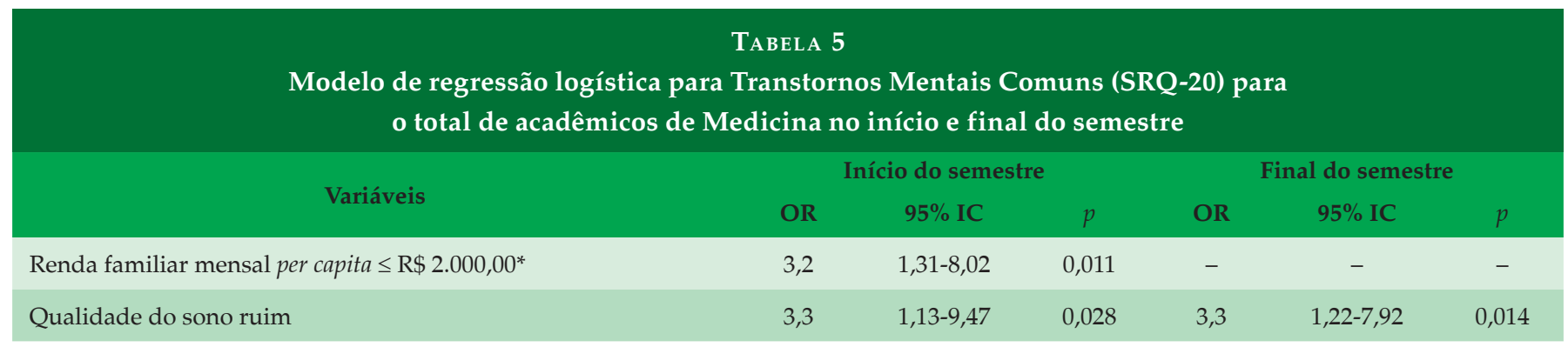

$O R=$ odds ratio

IC = intervalo de confiança.

* Equivalente a aproximadamente 3 salários mínimos ou US\$ 896,06 em 01/062013.

\section{DISCUSSÃO}

A prevalência de TMC encontrada neste estudo foi $35,8 \%$ no início e 51,5\% no final do semestre letivo. Prevalências menores de TMC avaliados pelo SRQ-20 foram encontradas na população brasileira, em relação às encontradas neste estudo, no início e final do semestre ${ }^{27-28}$ e no final do semestre ${ }^{29}$. Além disso, ambos os valores encontrados foram superiores às prevalências avaliadas pelo mesmo questionário, encontradas em estudos realizados com estudantes de Medicina do primeiro ao quarto ano no Vale do Paraíba $(26,1 \%)^{13}$; do segundo, quarto, sexto, oitavo, nono e décimo períodos em Porto Alegre $(29,6 \%)^{9}$; de todos os anos do curso na Universidade Federal da Paraíba $(33,6 \%)^{11}$; do segundo ao décimo segundo período na Universidade Federal de Sergipe $(42,5 \%)^{17}$ e em estudo sobre TMC e insônia $(22,19 \%)^{15}$.

A prevalência de 51,5\% de TMC encontrada neste estudo, no final do semestre letivo, foi superior aos $20 \%$ encontrados em estudantes de Medicina e aos 17,5\% em estudantes de Direito matriculados no terceiro, quarto e quinto ano, em pesquisa realizada entre maio e junho de 2001 em Pelotas, no Rio Grande do $\mathrm{Sul}^{16}$. Da mesma forma, foi superior aos $37,1 \%$ encontrados em uma avaliação realizada no final do ano letivo na Universidade Federal do Espírito Santo ${ }^{12}$, aos 44,7\% em avaliação realizada no final do semestre em Botucatu, em São Paulo ${ }^{10}$, e aos 44,9\% encontrados em estudantes também de Botucatu, em análise sobre apoio social e $\mathrm{TMC}^{18}$. Esses estudos foram realizados com estudantes dos seis anos do curso de Medicina e utilizaram o mesmo questionário citado acima para avaliar TMC.

Considerando os dados apresentados, as prevalências de TMC tanto no início como no final do semestre encontradas neste estudo, com diferença significativa, podem ser consideradas elevadas, além do aumento ocorrido ao longo do semestre, sendo que mais da metade do total de alunos no final do semestre eram possíveis casos de TMC. Deve-se levar em consideração que no final do semestre geralmente há maior cobrança dos alunos, com realização de provas e entrega de trabalhos, em comparação ao início do semestre, o que pode ter influenciado esse aumento da prevalência de TMC.

Não foi observada alteração da prevalência de TMC entre os anos do curso, tanto no início como no final do período. Sobre esse assunto, há diferentes achados na literatura. Alguns estudos realizados com estudantes de Medicina relataram maior número de casos no segundo e no quarto ano do curso $^{12}$ ou entre os alunos do curso básico e do clínico em relação aos alunos do internato ${ }^{11}$, enquanto um encontrou menor prevalência na primeira série $\mathrm{e}^{13} \mathrm{e}$ outro não observou diferenças entre os semestres avaliados ${ }^{9}$. Em um estudo, apesar de o período do curso não permanecer no modelo final de regressão logística, essa variável mostrou-se com tendência de risco para TMC ao longo do curso, sendo que o quinto período (início do ciclo profissional), o nono período (antes de começar o internato) e o internato pareceram ser os mais problemáticos ${ }^{17}$.

Diferenças relacionadas às características do processo de ensino-aprendizagem durante o curso e dos currículos de cada instituição onde as pesquisas foram realizadas podem ter influenciado os resultados, bem como diferenças regionais ou limitações metodológicas relativas aos delineamentos utilizados, que podem não reconhecer mudanças entre as diferentes turmas ao longo do tempo. Neste estudo, é importante considerar o pequeno tamanho das amostras na comparação entre cada turma, o que pode ter repercutido em não se ter observado diferenças nas prevalências de TMC.

Com relação ao questionário SRQ-20, a questão sobre sentir-se nervoso, tenso ou preocupado, na categoria de sintomas humor depressivo/ansioso, foi a que apresentou o maior percentual de respostas positivas, tanto no início como no final do semestre (71,6\% e 78,4\%, respectivamente). Essa questão também foi a que apresentou maior percentual de respostas positivas $(64,6 \%)$ em estudo sobre TMC em pro- 
fissionais de saúde de um hospital universitário ${ }^{30}$ e em residentes médicos e da área multiprofissional (73\%), ambos utilizando o SRQ-20 $0^{31}$.

Do ponto de vista acadêmico, é importante avaliar e acompanhar os efeitos do processo de ensino-aprendizagem sobre a saúde dos alunos e buscar estratégias para aliviar o sofrimento psicológico, que afeta o desempenho acadêmico, o desenvolvimento profissional e o desenvolvimento geral individual $^{6}$. Alunos com distúrbios emocionais e disfunções acadêmicas deveriam ser reconhecidos em um estágio inicial, sendo fundamental a disponibilidade de acesso a programas de saúde mental que prestem assistência formal, estruturada e confidencial ${ }^{32}$, seguindo o exemplo de importantes universidades brasileiras que já desenvolvem esses programas de ajuda psicoterapêutica ${ }^{33-34}$.

Apesar de algumas variáveis apresentarem significância estatística na análise bivariada para possíveis casos de TMC, na análise multivariada apenas duas permaneceram no modelo de regressão. Os fatores associados aos TMC no início do semestre foram renda familiar mensal per capita $\leq \mathrm{R} \$ 2.000,00 \mathrm{e}$ qualidade de sono ruim. Para o final do semestre, o único fator associado foi a qualidade do sono ruim.

Embora a variável sexo feminino tenha apresentado correlação com TMC no final do semestre na análise bivariada, essa variável não permaneceu significativa na análise multivariada. Assim como neste estudo, outros também não encontraram correlação significativa entre TMC e variáveis socioeconômicas e demográficas em estudantes de Medicina, como idade $^{9-10}$, sexo s-11,15,18$^{\text {, estado civil }}{ }^{9,18}$ e com quem reside ${ }^{10-11,18}$.

A renda familiar mensal per capita $\leq \mathrm{R} \$ 2.000,00$ foi um fator associado a possíveis casos de TMC no início do semestre. Em uma coorte populacional realizada no Sul do Brasil, uma prevalência maior de TMC entre os grupos de baixa renda e minorias étnico-raciais mostrou que as desigualdades sociais presentes no nascimento têm impacto importante sobre a saúde mental e transtornos mentais, especialmente comuns ${ }^{28}$. Em estudo transversal com adultos em Pernambuco, indivíduos com pior situação de renda familiar per capita apresentaram maior prevalência de $\mathrm{TMC}^{29}$.

Os "problemas financeiros" aparecem como a terceira causa $(32,4 \%)$ mais citada em pesquisa realizada para determinar os fatores de estresse em estudantes de Medicina ${ }^{7}$. De modo geral, menor renda pode ser fonte de preocupação para os estudantes, resultando em maiores dificuldades para a subsistência básica, aquisição de material para estudo e recursos para lazer, entre outros. Para os alunos do primeiro ano, o ingresso na universidade ocorre em uma fase de transição da adolescência para a vida adulta, em geral com muitas dúvidas e incertezas, exigindo dos estudantes um período de adaptação e mudança de estilo de vida ${ }^{35}$.

Também precisa ser considerado o fato de as turmas que participaram da pesquisa terem ingressado na universidade pelos sistemas de cotas. Sabe-se que o Brasil apresenta importantes desigualdades, sendo que as diferenças existentes nos níveis de educação e renda entre brancos e negros vão se disseminando de geração em geração. Os indicadores mostram que os negros, dentre outras variáveis, apresentam menores salários, desemprego maior e menor presença nas universidades quando comparados aos brancos ${ }^{36}$. Assim, desde 2002, os programas de expansão do ensino superior no País passaram a se vincular a políticas de inclusão racial e social, com o objetivo de reduzir as históricas defasagens na presença de negros e pobres nas instituições de ensino superior brasileiras, principalmente as públicas ${ }^{37}$. Na universidade onde foi realizada esta pesquisa, as vagas para ingresso nos cursos de graduação são distribuídas pelo sistema de cotas universal, de estudantes oriundos das escolas públicas e de estudantes negros oriundos das escolas públicas, o que talvez tenha influenciado esses resultados, como pode ser observado pela grande variabilidade da renda familiar per capita apresentada pelos estudantes.

A qualidade ruim do sono também foi um fator associado aos TMC. Os estudantes com qualidade de sono ruim neste estudo apresentaram uma chance 3,3 vezes maior de serem um possível caso de TMC, comparados aos que apresentavam boa qualidade de sono, tanto no início como no final do semestre. De modo semelhante, uma alteração no padrão do sono representou um risco 6,690 vezes maior para TMC em acadêmicos de Medicina 9 . O sono é geralmente considerado um fator para uma boa saúde e equilíbrio pessoal. Por outro lado, distúrbios do sono estão associados a faltas ao trabalho, ansiedade e depressã $0^{38}$, sendo que estudantes de Medicina são mais susceptíveis aos transtornos do sono devido à rotina acadêmica33. Dificuldade para iniciar o sono, dificuldade para manter o sono, dormir tarde e levantar cedo foram as variáveis associadas à possibilidade de transtorno psiquiátrico em 302 discentes da área médica ${ }^{15}$. Estudantes de Medicina são descritos na literatura como integrantes de um grupo de risco para o desenvolvimento de distúrbios do sono ${ }^{3}$, e sabe-se que tais distúrbios podem comprometer a qualidade de vida da pessoa ${ }^{39}$.

Os resultados deste estudo reforçam a possível associação entre qualidade de sono ruim e possíveis casos de TMC, estivesse o estudante no início ou final do semestre letivo. $\mathrm{Ou}$ seja, a má qualidade do sono ao final do semestre poderia ser potencializada pelo processo crônico de sono ruim, que ficará evidente à medida que os outros elementos de pressão e estresse interagirem ao longo do tempo. Dessa forma, para mi- 
nimizar essas dificuldades, os docentes e coordenadores dos cursos de Medicina poderiam planejar as aulas e as atividades extracurriculares com certo padrão de horários e promover o desenvolvimento de programas preventivos que orientem os alunos sobre a importância da regularidade e das medidas de higiene do sono ${ }^{3}$. Por outro lado, a qualidade do sono ruim como um fator associado aos TMC no início do semestre, logo após as férias escolares, quando ainda não estavam sendo realizadas provas e sem o possível efeito cumulativo de atividades curriculares e extracurriculares, sugere que os estudantes podem apresentar distúrbios de sono motivados por outras causas.

Como um dos elementos contidos na própria definição de saúde da Organização Mundial da Saúde ${ }^{40}$, o bem-estar mental deve ser ressaltado e enfatizado na atenção e cuidado dos estudantes. No decorrer do curso médico, os alunos são expostos a diversas causas chamadas não evitáveis de estresse, como lidar com o sofrimento e a dor dos pacientes, e causas evitáveis de estresse, relacionadas à estrutura do curso de $\mathrm{Me}$ dicina e ao relacionamento interpessoal ${ }^{5}$. Como estratégias desenvolvidas por estudantes de Medicina para enfrentamento do estresse citam-se, dentre outras, a valorização dos relacionamentos interpessoais e de fenômenos do cotidiano, equilíbrio entre estudo e lazer, organização do tempo, cuidados com a saúde, alimentação e o sono, prática de atividade física, religiosidade, trabalhar a própria personalidade para lidar com situações adversas e procura por assistência psicológica ${ }^{41}$. Quando o aluno não consegue elaborar os conflitos gerados pelas dificuldades do curso médico, é importante que ele tenha acesso a assistência psicológica e acompanhamento psicopedagógico $^{13}$.

Este estudo apresentou limitações, como ter sido realizado em uma única instituição e os resultados não poderem ser generalizados. Os estudantes que já estavam em tratamento para transtornos mentais podem não ter sido detectados como possíveis casos de TMC. Além disso, como a análise dos fatores associados com TMC foi realizada para os dois momentos em separado, não foi possível a atribuição de causalidade para as associações encontradas. Apesar dessas limitações, os resultados podem contribuir para o melhor entendimento de TMC na população estudada.

Os pontos fortes deste estudo foram a possibilidade de comparar as prevalências de TMC no início e final do semestre em estudantes de Medicina e a identificação de possíveis fatores associados aos TMC. Além disso, outro aspecto importante foi o uso de questionários específicos, padronizados e internacionalmente validados.

\section{CONCLUSÃO}

Os resultados encontrados demonstraram alta prevalência de transtornos mentais comuns entre os estudantes de Medicina. Também se observou aumento na prevalência de TMC do início para o final do semestre. No início do semestre, os TMC foram independentemente relacionados com menor renda familiar mensal per capita e qualidade de sono ruim, e no final do semestre a qualidade de sono ruim. Essas informações são importantes para direcionar intervenções inovadoras de prevenção e de estratégias de suporte e enfrentamento quanto ao cuidado da saúde mental dos estudantes de Medicina, do ponto de vista tanto psicológico como pedagógico, visando melhorar sua qualidade de vida, auxiliar em suas necessidades durante a graduação e, assim, aprimorar sua formação profissional e pessoal.

\section{REFERÊNCIAS}

1. Saravanan C, Wilks R. Medical students' experience of and reaction to stress: the role of depression and anxiety. Scientific World Journal. 2014;29:73-82.

2. Pagnin D, Queiroz V. Comparison of Quality of Life Between Medical Students and Young General Populations. Educ Health. 2015;28(3): 209-12.

3. Cardoso HC, Bueno FCC, Alves APR, et al. Avaliação da qualidade do sono em estudantes de Medicina. Rev Bras Educ Med. 2009;33(3):349-55.

4. Enns MW, Cox BJ, Sareen J, Freeman P. Adaptive and maladaptive perfectionism in medical students: a longitudinal investigation. Med Educ. 2001;35(11):1034-42.

5. Costa CL, Pereira CA. O abuso como causa evitável entre estudantes de Medicina. Rev Bras Educ Med. 2005;29(3):185-90.

6. Facundes VLD, Ludemir AB. Common mental disorders among health care students. Rev Bras Psiquiatr. 2005;27:194-200.

7. Barikani A. Stress in Medical Students. J Med Education. 2008;11:41-4.

8. Radcliffe C, Lester H. Perceived stress during undergraduate medical training: a qualitative study. Med Educ. 2003;37(1):32-8.

9. Almeida AM, Godinho TM, Bitencourt AG, et al. Common mental disorders among medical students. J Bras Psiquiatr. 2007;56(4):245-51.

10. Lima MC, Domingues MS, Cerqueira AT. Prevalence and risk factors of common disorders among medical students. Rev Saude Publica. 2006;40:1035-41.

11. Rocha ES, Sassi AP. Transtornos mentais menores entre estudantes de Medicina. Rev Bras Educ Med. 2013;37(2):210-216. 
12. Fiorotti KP, Rossoni RR, Borges LH, Miranda AE. Transtornos mentais comuns entre estudantes do curso de Medicina: prevalência e fatores associados. J Bras Psiquiatr. 2010;59(1):17-23.

13. Cunha MAB, Neves AAF, Moreira ME, et al. Transtornos psiquiátricos menores e procura por cuidados em estudantes de Medicina. Rev Bras Educ Med. 2009;33(3):321-328

14. Benvegnu LA, Deitos F, Copette FR. Problemas psiquiátricos menores em estudantes de Medicina da Universidade Federal de Santa Maria, RS, Brasil. Rev Psiquiatr Rio Gd Sul. 1996;18:229-33.

15. Loayza HMP, Ponte TS, Carvalho CG, et al. Association between mental health screening by self-reporting questionnaire and insomnia in medical students. Arq Neuropsiquiatr. 2001;59(2-A):180-5.

16. Volcan SMA, Sousa PLR, Mari JJ, Horta BL. Relação entre bem-estar espiritual e transtornos psiquiátricos menores: estudo transversal. Rev Saúde Pública. 2003;37(4):440-5.

17. Costa, EFO, Andrade TM, Silvany Neto AM, Melo EV, Rosa ACA, Alencar MA et al . Common mental disorders among medical students at Universidade Federal de Sergipe: a cross-sectional study. Rev. Bras. Psiquiatr. 2010;32(1):11-9.

18. Silva AG, Cerqueira ATAR, Lima MCP. Apoio social e transtorno mental comum entre estudantes de Medicina. Rev Bras Epidemiol. 2014;17(1):229-42 .

19. Goldberg D, Huxley P. Common mental disorders: a bio-social model. Acta Psychiatr Scand Suppl. 1994;385:66-70

20. Mari JJ, Jorge MR. Transtornos psiquiátricos na clínica geral. Psychiatry On-line Brazil 1997;2. Disponível em: http:/ / www.polbr.med.br/ano97/tpqcm.php

21. Rocha SV, Almeida MMG, Araujo TM, Virtuoso Jr JS. Prevalence of common mental disorders among the residents of urban areas in Feira de Santana, Bahia. Rev Bras Epidemiol. 2010;13(4):1-11.

22. Harding TW, de Arango MV, Baltazar J, et al. Mental disorders in primary health care: a study of the frequency and diagnosis in four developing countries. Psychol Med. 1980; (10):231-41.

23. Mari JJ, Williams P. A validity study of a psychiatric screening questionnaire (SRQ-20) in primary care in the city of São Paulo. Br Psychiatry.1986;148:23-6.

24. Iacoponi E, Mari JJ. Reliability and factor structure of the Portuguese version of self-reporting questionnaire. Int J Soc Psychiatry. 1988;35(Suppl3):213-222.

25. Buysse DJ, Reynolds CF, Monk TH, et al. The Pittsburgh Sleep Quality Index: a new instrument for psychiatric practice research. Psychiatric Research. 1989;28:183-213.
26. Bertolazi AN, Fagondes SC, Hoff LS, et al. Validation of the Brazilian Portuguese version of the Pittsburgh Sleep Quality Index. Sleep Med. 2011;12(1):70-5.

27. Lima MS, Beria JU, Tomasi E, Conceicão AT, Mari JJ. Stressful life events and minor psychiatric disorders: an estimate of the population attributable fraction in a Brazilian community-based study. Int J Psychiatry Med. 1996;26(2):21122.

28. Anselmi L, Barros FC, Minten GC, Gigante DP, Horta BL, Victora CG. Prevalence and early determinants of common mental disorders in the 1982 birth cohort, Pelotas, Southern Brazil. Rev Saude Publica. 2008;4 Sup 2:26-33.

29. Ludermir AB, Melo Filho DA. Living conditions and occupational organization associated with common mental disorders. Rev Saude Publica. 2002;36(2):213-21.

30. Alves AP, Pedrosa APK, Coimbra MPR, et al. Prevalência de transtornos mentais comuns entre profissionais de saúde. Rev enferm UERJ. Rio de Janeiro. 2015;23(1):64-9.

31. Carvalho CN, Melo-Filho DA, Carvalho JAG, et al. Prevalência e fatores associados aos transtornos mentais comuns em residentes médicos e da area multiprofissional. J Bras Psiquiatr. 2013;62:38-45.

32. Nogueira-Martins LA, Fagnani Neto R, Macedo PC, Cítero VA, Mari JJ. The mental health of graduate students at the Federal University of São Paulo: a preliminary report. Braz J Med Biol Res. 2004;37(10):1519-24.

33. Nogueira-Martins LA, Stella RC, Nogueira HE. A pioneering experience in Brazil: the creation of a center for assistance and research for medical residents (NAPREME) at the Escola Paulista de Medicina, Federal University of São Paulo. Sao Paulo Med J. 1997;115(6):1570-4.

34. Millan LR, Arruda PC. Psychological assistance to medical students: 21 years of experience. Rev Assoc Med Bras. 2008;54(1):90-4.

35. Scherer ZAP, Scherer EA, Carvalho AMP. Reflexões sobre o ensino da enfermagem e os primeiros contatos do aluno com a profissão. Rev Latino-Am Emfermagem. 2006;14(2):285-91.

36. Haas CM, Linhares M. Políticas públicas de ações afirmativas para ingresso na educação superior se justificam no Brasil? Rev. Bras. Estud Pedagog. 2012;93(235):836-63.

37. Cervi EU. Ações afirmativas no vestibular da UFPR entre 2005 a 2012: de política afirmativa racial a política afirmativa de gênero. Rev Bras Ciênc Polít. 2013;11:63-88.

38. Chan-Chee C, Bayon V, Bloch J, et al. Epidemiology of insomnia in France. Rev Epidemiol Sante Publique. 2011 59(6):409-22. 
39. Muller MR, Guimarães SS. Impacto dos transtornos do sono sobre o funcionamento diário e a qualidade de vida. Estud Psicol (Campinas). 2007;24(4):519-28.

40. Organização Mundial da Saúde (OMS). Relatório mundial da saúde. Saúde mental: nova concepção, nova esperança. Lisboa; 2001.

41. Zonta R, Robles ACCM, Grosserman S. Estratégias de enfrentamento do estresse desenvolvidas por estudantes de Medicina da Universidade Federal de Santa Catarina. Rev Bras Educ Med. 2006;30(3):147-53.

\section{CONTRIBUIÇÃO DOS AUTORES}

Carlos Magno Guimarães Ferreira: Participou da concepção do estudo, coleta, análise e interpretação dos dados, redação do texto e revisão da versão final do texto. Ana Claudia Gara- beli Cavalli Kluthcovsky: Participou da concepção do estudo, análise e interpretação dos dados, redação do texto e revisão da versão final do texto. Tatiana Menezes Garcia Cordeiro: Participou da concepção do estudo, interpretação dos dados e revisão da versão final do texto.

\section{CONFLITO DE INTERESSES}

Os autores declaram não haver conflito de interesses.

\section{ENDEREÇO PARA CORRESPONDÊNCIA}

Carlos Magno Guimarães Ferreira

Rua Engenheiro Schamber, 160, ap. 54

Centro - Ponta Grossa

CEP: 84010-340 PR

Email: carlosmagnogf@gmail.com 